\title{
Independent segmental bone transport of the radius and ulna: $A$ case report
}

\author{
FF Birkholtz ${ }^{1,2}$ MBChB, MMed(Orth), FCOrth(SA) \\ P Greyling ${ }^{1}$ MBChB(Pret), MMed(Orth)(Pret), FCS(Orth)SA \\ ${ }^{1}$ Department of Orthopaedics, University of Pretoria \\ ${ }^{2}$ Walk-A-Mile Centre for Advanced Orthopaedics, Centurion, Pretoria \\ Correspondence: \\ Dr Franz F Birkholtz \\ Tel: 0126442641 \\ Fax: 0126442642 \\ Email: franz.birkholtz@walkamile.co.za \\ Address: PO Box 11328, Centurion, 0046
}

\begin{abstract}
Significant bone loss in the forearm involving both the radius and ulna is a difficult problem to manage. The functional outcome may be limited due to loss of pro- and supination.

We present a case where significant bone loss of both the radius and the ulna in an adult patient was successfully reconstructed using segmental bone transport. The radius and ulna were transported independently from each other to try and maintain function. A reasonable functional result was achieved.
\end{abstract}

Key words: bone transport, distraction osteogenesis, forearm bone loss, radius ulna transport, cement spacer

http:/ / dx.doi.org/10.17159/2309-8309/2016/v15n2a4

\section{Introduction}

Significant segmental bone loss in the forearm is a difficult clinical entity to treat. Strategies described in literature include interposition grafting, double-barrel free fibula reconstruction, induced membrane techniques, and converting to a single bone forearm by establishing a crossunion.

Although the continuity of bone can be restored, the complex relationship of the radius and ulna may be disturbed, leading to a limitation of pro- and supination.

Reconstructing the radius and ulna independently with distraction osteogenesis bone transport has the theoretical advantage of maintaining the complex relationship between the radius and ulna in the forearm. In principle this should provide the ability to maintain a measure of pro- and supination after reconstruction.

\section{Case report}

A 34-year-old woman was involved in a motor vehicle accident. She was a right-handed social worker at the time of injury and was systemically healthy. The injury was limited to the right upper limb, where she sustained severely comminuted fractures of the right radius and ulna. Figure 1 shows the initial radiographic appearance. There was a large dorsal wound overlying the distal forearm region, measuring $5 \times 8 \mathrm{~cm}$.

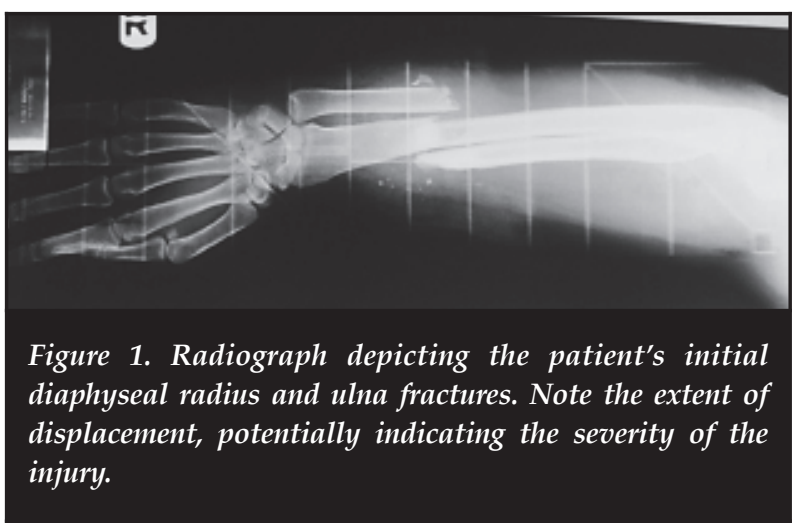

Initially the patient presented to a regional hospital, where a primary debridement was performed and intravenous antibiotic therapy was instituted. An open reduction and internal fixation was performed with plates and screws. Shortly afterwards she developed a fulminant infection in the fracture sites. She was splinted and referred to our unit for further management.

A thorough debridement and removal of instrumentation was performed and the radius and ulna were fixed with a temporary modular bar-to-pin external fixator. Three days later a wound inspection was carried out and approximately $5 \mathrm{~cm}$ of necrotic bone was removed from the radius and ulna. 
An antibiotic cement spacer with gentamycin was created using off-the-shelf bone cement and inserted into the bone defect. Broad-spectrum intravenous antibiotics were administered. Figure 2 shows the antibiotic cement spacer in situ, as well as the stabilisation using the modular barto-pin external fixator.

The patient was referred to the plastic and reconstructive surgeon who provided durable soft tissue cover with a radial forearm flap.

Six weeks later the temporary external fixator and polymethylmetacrylate cement spacer were removed. A paediatric monorail fixator (LRS, Orthofix, Verona, Italy) was applied with six hydroxyapatite-coated half pins on each of the two forearm bones as depicted in Figure 3. A predrilled osteotomy was done proximally for bone transport in both the radius and ulna. After a waiting period of 10 days, distraction progressed at a rate of $1 \mathrm{~mm}$ per day. Throughout the distraction period, the patient was seen by the rehabilitation team and progressed with intensive hand therapy to maintain joint mobility and hand function.

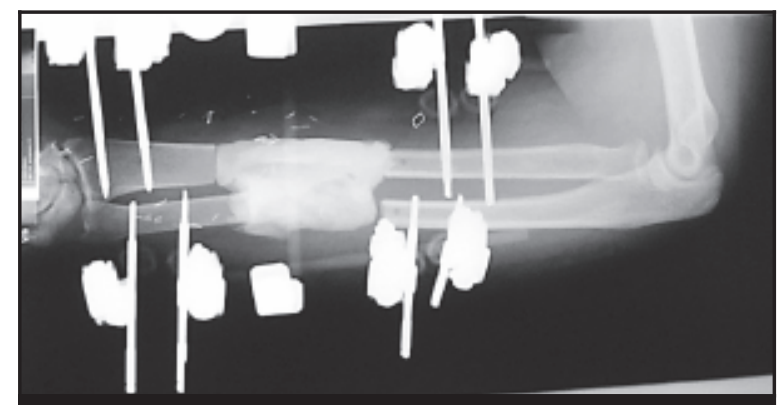

Figure 2. Radiograph depicting stabilisation of the radius and ulna with a modular pin-to-bar external fixator. Also note the antibiotic bone cement spacer placed in the defect of the radius and ulna.

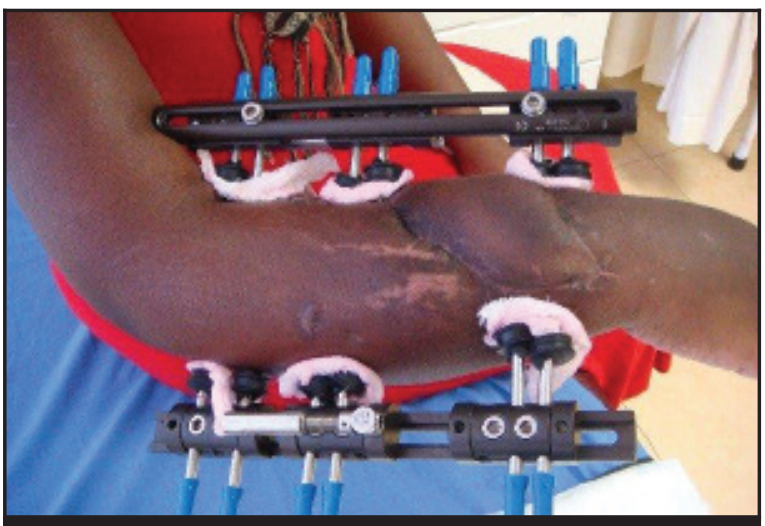

Figure 3. A clinical photograph showing the appearance of the two external fixators on the forearm. Note also the presence of the radial forearm flap used to provide durable soft tissue cover.

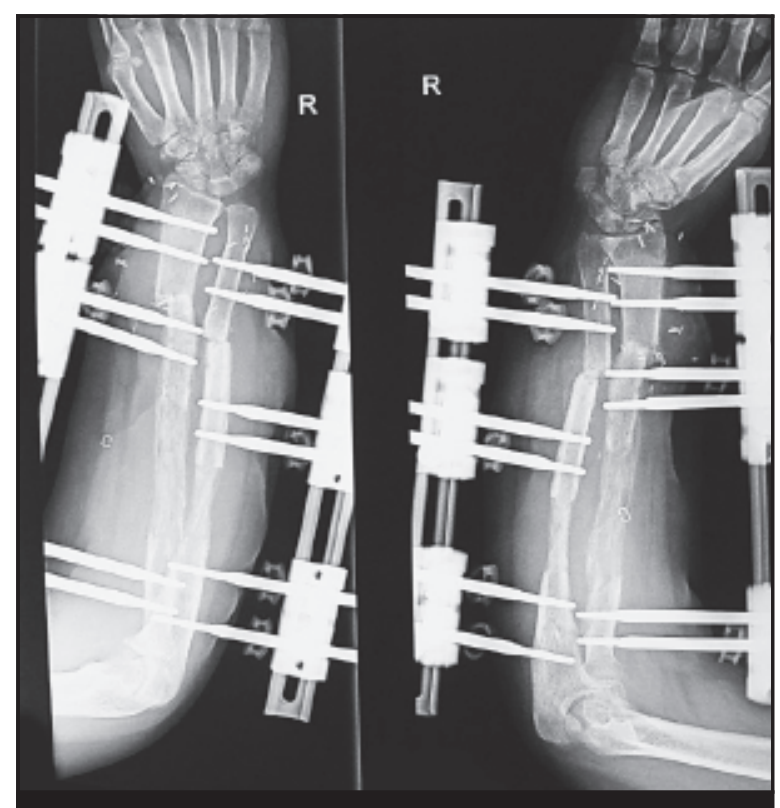

Figure 4. Radiograph depicting the forearm after distraction osteogenesis and subsequent open docking of the radius and ulna.

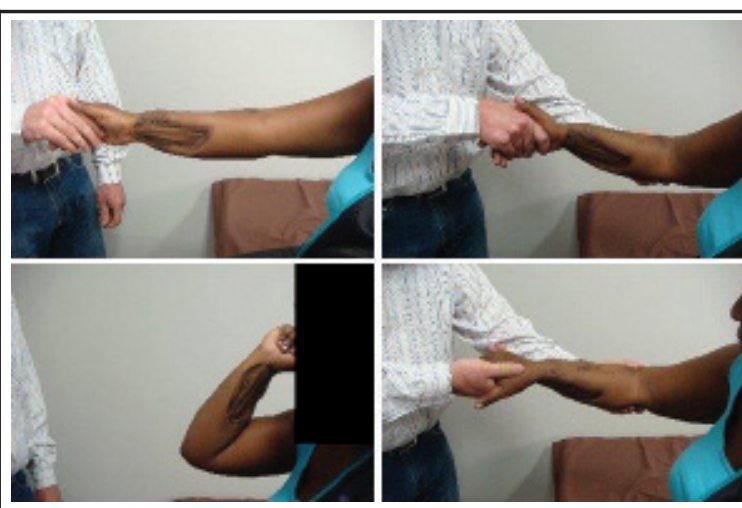

Figure 5. These clinical pictures were taken at final follow-up 4 years after the initial treatment and show a reasonable range of motion of the forearm.

Once distraction was complete and the bone defects obliterated by distraction osteogenesis, an open docking procedure was performed on both the radius and ulna. During this procedure, the bone ends at the docking site were freshened, interpositional fibrosis removed and the fragments compressed acutely using the external fixator. The docking site was augmented with cancellous autograft obtained from the right proximal tibia. Figure 4 demonstrates the radiographic appearance after the docking procedure.

Approximately three months after docking, the LRS external fixators were removed and locking nails inserted into the radius and ulna. The ulna docking site was regrafted. Final time in external fixator was 142 days which represents an external fixation index of just under 1 month per $\mathrm{cm}$ of bone lengthening. 
Two months later the fractures were radiologically and clinically united.

The patient was followed up to 4 years after the index procedure and has returned to her prior occupation. She reported no chronic pain and has shown no evidence of infection. At final assessment she had an elbow arc of motion from 0 to 140 degrees of flexion. She was able to supinate to neutral and had pronation of up to 60 degrees (Figure 5).

\section{Discussion}

The special anatomical relationship between the radius and ulna require anatomical reduction to restore bone stability and function of forearm pro- and supination. The radius and ulna function as a unit together with the proximal and distal joints and is viewed as a bicondylar joint. For these reasons diaphyseal forearm fractures should be viewed as intraarticular lesions. ${ }^{1}$

Most reconstruction techniques have been described for the lower limb. Literature on treating forearm bone loss is limited to a small series of case studies. Techniques that have been described to treat forearm bone loss include interposition grafting, double barrel free fibula reconstruction, induced membrane techniques, segmental bone transport and the creation of a single bone forearm by establishing a cross-union. ${ }^{2,3}$

These techniques do not replace the need for a thorough debridement and excision of necrotic and infected bone and tissue. Infection is not healed by the simple compression of the fracture site alone..$^{2-4}$

When dealing with forearm non-unions and bone loss, soft tissue adhesions and scar tissue need to be addressed. ${ }^{4}$ As was the case in this instance, meticulous attention to rehabilitation is needed to optimise the functional outcome.

A cement spacer was used to manage the transport space. Because it was preloaded with antibiotics, it provided an added benefit of local infection control. Cement spacers have the added benefit of membrane induction, which leads to a bio-active pseudo-periosteum which can aid in bone reconstruction.

Durable soft tissue coverage is an important determinant in successful segmental bone transport. ${ }^{2}$ In this case it was provided by a radial forearm flap, which is a pliable, lowprofile fasciocutaneous flap.

The monolateral frames used in this case provided the advantage of independently but concurrently reconstructing each of the two bones. The intention was to try and preserve the ellipse of the forearm and to preserve elbow range of motion in flexion, extension, pronation and supination. Based on the range of motion at final assessment at 4 years' follow-up, it seems that this philosophy was, in part, successful.

An open docking procedure with an autologous bone graft at the docking site is in accordance with the data reported in the literature and important to prevent repeated fractures and nonunion. ${ }^{2,5}$
Intra-medullary fixation provided stability maintaining the radius and ulna length, while still allowing the radius and ulna to function individually. ${ }^{3}$ In this instance it also provided internal stability to the tenuous regenerates and docking sites to facilitate rehabilitation and earlier external fixator removal.

\section{Summary}

Significant bone loss in the forearm involving both the radius and ulna is a difficult problem to manage. The functional outcome may be limited due to loss of pronation, supination and grip strength. This case report illustrates a novel surgical approach to independently transport the radius and ulna, and thus provide the best possible functional outcome in this devastating condition.

\section{Compliance with Ethics Guidelines}

The content of the article is the sole work of the authors. FF Birkholtz and P Greyling declare that no benefits of any form have been or are to be received from a commercial party related directly or indirectly to the subject of the article. The procedures followed were in accordance with the ethical standards of the Helsinki Declaration (1964, amended most recently in 2008) of the World Medical Association. The patient's written consent was obtained for inclusion of her data in this paper.

\section{References}

1. Jupiter JB, Fernandez DL, Levin SL, Wysicki RW. Reconstruction of Posttraumatic Disorders of the Forearm. J Bone Joint Surg Am 2009;91:2730-39.

2. Rigal S, Merloz P, Le Nen D, Mathevon H, Masquelet AC, the French Society of Orthopaedic Surgery and Traumatology (SoFCOT). Bone transport techniques in posttraumatic bone defects. Orthopaedics and Traumatology: Surgery and Research 2012;98:103-108.

3. Gupta DK, Kumar G. Gap nonunion of forearm bones treated by modified Nicoll's technique. Indian J Orthop 2010;44(1):84-88.

4. Liu T, Liu Z, Ling L, Zhang X. Infected forearm nonunion treated by bone transport after debridement. BMC Musculoskeletal Disorders 2013;14:273.

5. Chunshen W, Zhaohua B, Chenxi Y, Cheng C, Huilin Y, Jun $Z$. Bone transport combined with locking plate and bone grafting for treatment of nonunion of the ulna: a case report. Int J Clin Exp Med 2013;6(10):996-1000.

This article is also available online on the SAOA website (www.saoa.org.za) and the SciELO website (www.scielo.org.za). Follow the directions on the Contents page of this journal to access it. 